\title{
A simple and rapid method for generating patterned co-cultures with stable interfaces
}

\author{
Sahar Javaherian ${ }^{1, *}$, Katherine J. Li ${ }^{1, *}$, and Alison P. McGuigan ${ }^{1,2}$ \\ ${ }^{1}$ Department of Chemical Engineering and Applied Chemistry, and Institute of Biomaterials and Biomedical \\ Engineering, University of Toronto, Toronto, Canada \\ \#S.J. and K.J.L. contributed equally to this work.
}

BioTechniques 55:21-26 (July 2013) doi 10.2144/000114051

Keywords: cell patterning; co-culture, tissue boundary; tissue engineering; in vitro screening

Supplementary material for this article is available at www.BioTechniques.com/article/114051.

In native tissues, different cell types are organized into defined structures and architectures that are critical for correct tissue function. In vitro cellular patterning methods enable control over the spatial organization of cells, permitting, to some extent, the reproduction of native tissue structures and the generation of a more "in vivo-like" culture platform. While this is advantageous for applications such as drug screening, existing patterning methods are time-consuming, labor-intensive, and low-throughput. Here, we describe a novel medium-throughput patterning strategy for generating spatially controlled co-cultures of two cell types based on differential deposition of BSA solution in a tilted plate. Our method allows generation of homotypic and heterotypic co-cultures that are stable for at least seven days in culture. The reproducibility and consistency of this patterning technique, together with its low cost and ease of use, make it a promising cell culture platform for medium- to high-throughput screening using high-content imaging.

Appropriate cellular organization is vital for healthy tissue function. Consequently, structural disruption within tissues is a hallmark of many diseases $(1,2)$. Patterned co-cultures of cells can be used to study interactions between different cell types and the effect of cell organization on tissue function in vitro. Cellular patterning is possible using a set of techniques that control cell localization on a culture substrate in vitro (3-5). In patterned co-cultures, the localization of different cell types is controlled, allowing for the generation of "tissue" with a pre-defined composition and architecture. There are typically two approaches to generating co-cultures: $(i)$ micropatterning, which allows direct cellcell contact, as occurs in vivo, and (ii) coculture in a multi-compartment system, such as a transwell plate, that physically separates the two cell populations. While the latter approach is easier, the interaction between different cell types is limited to diffusible signals. Direct cell-to-cell contact produces significantly different cell behaviors from those observed in multi-compartment co-culture (6-8). For this reason, methods to produce direct contact co-cultures may provide more relevant in vitro models for understanding cell and tissue function. Such in vitro models could be particularly useful for screening applications. Indeed, integration of direct coculture patterning techniques into current high-throughput drug screening protocols is predicted to increase the success rate of drug discovery by promoting a more in vivo-like cell culture system (9). More specifically, patterned co-cultures can be valuable systems for screening changes in cell-cell interactions. For example, screens of patterned co-cultures could be used to search for compounds that inhibit infiltration of tumor cells into sheets of other cell types. Another example is in regenerative therapy, where infiltration of therapeutic cells into damaged tissue is necessary for regeneration (10). Despite the promise of direct contact co-culture, there are currently no medium- to high-throughput methods for generating patterned 2-D co-cultures.
Most current co-culture patterning techniques involve the use of manual stamps $(11,12)$ or stencils $(13,14)$ to prevent cell attachment to certain locations on the substrate. Significant optimization is required to generate reliable and consistent patterning using these methods. Several attempts have been made to adapt existing patterning strategies for higher throughput. For example, microstamping techniques allow stamping of multiple well surfaces in parallel (15). While this method could potentially be adapted to generate patterned co-cultures, in its current form it is only suitable for generation of patterned mono-cultures. Moreover, this method is difficult to optimize and currently only allows 6 to 12 wells in a 96-well plate to be patterned in parallel. An alternative is high-throughput patterning using microarray spotting technologies $(16,17)$. This approach allows high control and precision, but is labor-intensive and requires specialized equipment. Moreover, the use of microarray spotting for patterning direct contact cellular

\section{Method summary:}

Our method allows the generation of simple, spatially controlled co-cultures of two cell types based on differential deposition of BSA in a tilted multi-well plate. The procedure is simple, allows medium- to high-throughput pattern generation, is compatible with high-content microscopy, and allows patterning of homotypic and heterotypic co-cultures that are stable for at least seven days. 
co-cultures remains to be demonstrated. Microfluidic methods are also available, but these $(18,19)$ are often complex to fabricate, can compromise cell viability during extended culture, and are not easily compatible with high-throughput screening methods such as high-content microscopy.

Here we describe a simple method for generating patterned co-cultures with predictable cell organization that is suitable for mediumto high-throughput applications. Our method allows limited spatial control over the localization of two cell types in multi-well plates and is compatible with high-content screening platforms. The ratio between cell types in each well can be varied on demand, which is useful for probing the effect of diffusible signals in combination with direct cell-cell contact. Cell patterning is controlled by sequential seeding of two cell populations onto substrates using selective deposition of cell-repellent protein (BSA). This is achieved by placing a set volume of BSA solution in the well while tilting the plate at a specific angle. We demonstrate the versatility of our co-culture method by generating co-cultures of various cell combinations in wells of different sizes. Further, we characterize the reproducibility and consistency of our patterning technology in order to confirm its compatibility with automated high-content imaging.

\section{Materials and methods}

\section{Cell culture}

We used the ARPE-19 human retinal epithelial cell line, the MDCK dog kidney epithelial cell line, the NIH $3 \mathrm{~T} 3$ and BJ (ATTC, Manassas, VA) fibroblast cell lines at passages P10-P20, and human umbilical vein endothelial cells (HUVECs) (Lonza, Basel, Switzerland) at passages P4-P8 grown in medium recommended by the supplier. Populations of eGFP (green fluorescing) or mCherry (red fluorescing) overexpressing cells were prepared by lentivirus transduction. The eGFP lentiviral construct was a gift from J Axelrod at Stanford and we cloned in the mCherry gene (Clontech, Mountain View, CA, USA) into the backbone to generate the mCherry lentiviral construct.

\section{Plate pre-patterning with BSA}

To generate pre-patterned plates with differential BSA deposition, we added $200 \mu \mathrm{L}$ of $0.5 \%$ w/v BSA (Sigma Aldrich, Oakville, Ontario, Canada) to cover only the bottom half of each well in a 24-well tissue-culture treated plate (BD Biosciences, Mississauga, Ontario, Canada). The plate was tilted using a stand and incubated for $2 \mathrm{~h}$ at $37^{\circ} \mathrm{C}$ and $5 \% \mathrm{CO}_{2}$. For 6-, 12-, 48-, and 96-well plates, $800,400,100$, and $50 \mu \mathrm{l} \mathrm{BSA}$ was added to each well respectively. After incubation, the BSA solution was removed carefully and the plate was air-dried to ensure that half the well remained BSA-free.

\section{Cell patterning}

The first cell type to be seeded was suspended in serum-free medium (DMEM-F12; Gibco, Gaithersburg, MD), seeded on the plate, and incubated. The seeding densities, volumes, and incubation times for different well sizes are listed in Table 1. After gently removing excess cells, we covered the entire well with $500 \mu \mathrm{L}$ of $10 \mu \mathrm{g} / \mathrm{mL}$ fibronectin (Biomedical Techonologies Inc., Stoughton, MA) and incubated the plate for $30 \mathrm{~min}$. We suspended the second cell type in complete medium at a density of $2 \times 10^{6}$ cells $/ \mathrm{mL}$. The fibronectin solution was removed and $500 \mu \mathrm{L}$ (for a 24-well plate) of the second cell type suspension was added. The plate was incubated for 3-4 h until the cells were attached before washing off unadhered cells to reveal a co-culture with a robust interface.

\section{Microscopy and image analysis}

Co-cultures were imaged either shortly after generation (4-12 h) or maintained in their culture medium for 7 days, with medium exchange every 2 days, and then imaged. The fluorescent images were taken with an ImageXpress Micro Microscope (Molecular Devices, Sunnyvale, CA) at $4 \times$ magnification. For the purpose of visualizing the whole well, multiple images at $4 \times$ were acquired and stitched together using MetaExpress software (Molecular Devices).

To quantify the reproducibility of patterning, each well in a 24-well plate was patterned with ARPE-19-GFP cells as described above. Images of each well (the entire well) were acquired. We analyzed the location of the pattern interface by measuring they-coordinate of the interface at different $\mathrm{x}$-coordinates. The coordinates were then converted into distance from the bottom left corner of the screen (for clarity of presentation) and used to calculate mean and standard deviation.

\section{Results and discussion}

Figure 1A shows a schematic of our approach for generating patterned co-cultures in multiwell plates based on differential deposition of BSA as well as the outcome of each step of the patterning process. BSA is a commonly used protein for blocking the adsorption of other cell-adhesive proteins, thus preventing both specific and non-specific binding of cells to a culture surface $(20,21)$. The first critical step in the patterning process is differential deposition of BSA on the tissue culture polystyrene (TCPS) surface. To achieve this, we placed a multi-well plate (24-well size in this case) on a slanted support angled at approximately $40^{\circ}$ and added $200 \mu \mathrm{L}$ of $0.5 \%$ BSA solution to each well using a multi-channel pipettor (Figure 1B and C). This volume was chosen to ensure that approximately half of the well was pre-patterned with BSA. The profile of the BSA solution in the angled well ultimately dictates the profile of the cellular patterns generated. We found that by controlling the angle under which the plate is placed (Supplementary FigureS1) or the amount of BSA solution placed into each well (Supplementary Figure S2), we could control the ratio of the well surface that was covered with BSA and, ultimately, the space available for cell type 2 .

Plates were incubated for 2 hours in a slanted position at $37^{\circ} \mathrm{C}$ to allow BSA deposition onto the polystyrene surface. It was important to keep the angle of the plate constant at all times. After the incubation, we aspirated BSA from the wells and let them air dry. Successful BSA deposition and the subsequent establishment of a clear boundary between BSA-exposed and non-exposed regions are critical for the outcome of patterning. Conveniently, we also found that we were able to generate patterned co-cultures using BSA-coated plates prepared up to three weeks prior to patterning if stored in a dry place at room temperature, making this patterning method even more attractive for screening applications.

Following preparation of the plates, we seeded a suspension of cell type 1 in serum-free medium into normally oriented (non-slanted) plates. The use of serum-free medium was critical for our differential adhesion strategy to work robustly: serum or other proteins in the medium can adsorb on the substrate and mask the blocking effect of $\mathrm{BSA}$, resulting in incorrect patterning. The number of cells added during this step was also crucial. The total amount of cells seeded should cover $100 \%$ of the well surface (Figure 1D). For most cell types tested, $2 \times 10^{6}$ cells suspended in $500 \mu \mathrm{L}$ provided optimal results for seeding a 24-well plate. If the cell density is too low, cell type 1 does not generate a confluent sheet and cell type 2 will adhere to unwanted areas, resulting in disrupted cell adhesion patterns. Seeding densities may require adjustment depending on cell size.

Over time, cells seeded in the pre-patterned wells attached only to the BSA-free regions of the substrate. However, the duration of this

Table 1. Patterning conditions for wells of different sizes.
\begin{tabular}{|l|l|l|c|}
\hline Well size & BSA concentration, \% w/v & Number of cells seeded & Incubation time \\
\hline 6-well & 0.5 & $4 \times 10^{6}$ & $105 \mathrm{~min}$ \\
\hline 12-well & 0.5 & $2 \times 10^{6}$ & $105 \mathrm{~min}$ \\
\hline 24-well & 0.5 & $1 \times 10^{6}$ & $90 \mathrm{~min}$ \\
\hline 48-well & 0.5 & $5 \times 10^{5}$ & $75 \mathrm{~min}$ \\
\hline 96-well & 0.75 & $2.5 \times 10^{5}$ & $35 \mathrm{~min}$ \\
\hline
\end{tabular}



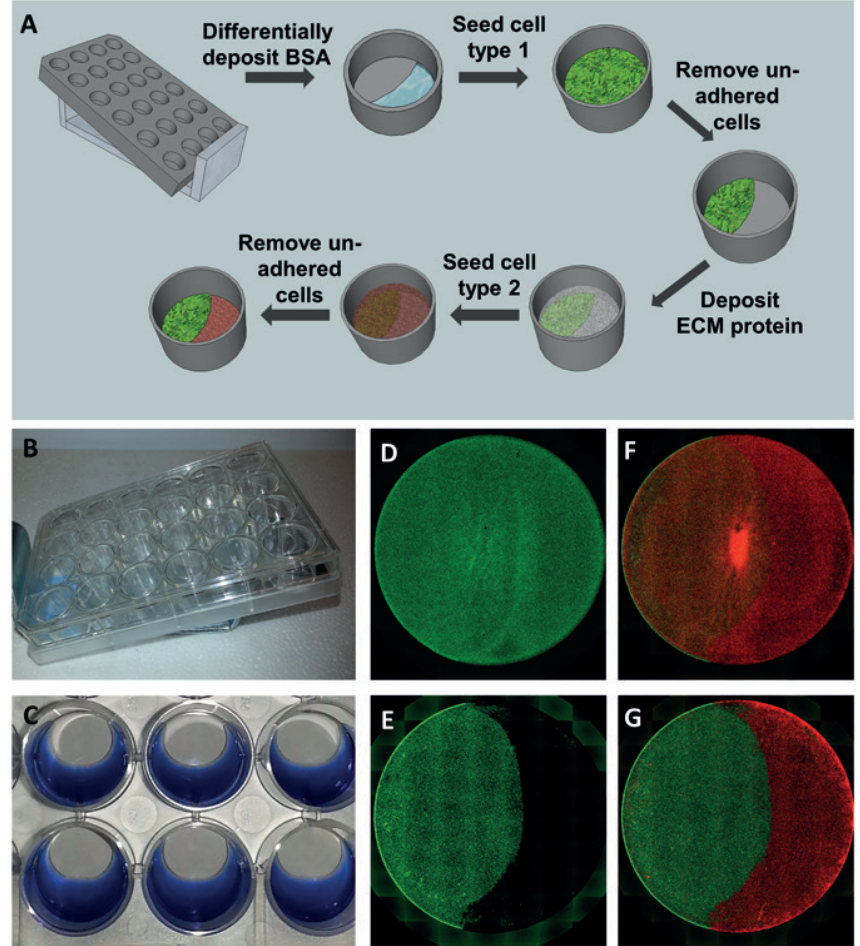

Figure 1. Co-culture patterning process. (A) Schematic of patterning strategy. The process is demonstrated in a 24-well plate. (B) A multi-well plate is placed on a slant. (C) BSA solution (colored with blue food coloring for demonstration purposes) is placed in the wells of the slanted plate so that only a portion of the surface is covered. (D) Cell type 1 (GFP-ARPE-19; green) is seeded so that $100 \%$ of the surface is covered with cells. (E) After a $1.5 \mathrm{~h}$ incubation, non-adhered cells are removed by washing, resulting in a patterned interface. $(F)$ Cell type 2 (mCherryARPE-19; red) is seeded so that $100 \%$ of the surface is covered with cells. (G) Non-adhered cells are removed by washing, producing a patterned co-culture of the two cell types. D, E ,F, and G are views of an entire well of a 24-well plate generated by stitching together multiple images taken at a magnification of $4 \times$.

incubation step is important for successful patterning. Sufficient time must be allowed for the cells to attach and spread on the BSA-free part of the plate, but extended incubations can result in undesirable cell attachment to BSA-covered regions. Once the cells were attached to the BSA-free region, we removed non-adhered cells by washing the plate 3-5 times with serum-free medium. This step produced a clear interface between the region where cell type 1 is attached and the cell-free region (Figure 1E). We also found that we could achieve cell patterning by tilting a solution of fibronectin in non-tissue culture treated polystyrene plates (Supplementary Figure S3). However, because the cost of BSA is significantly lower than that of fibronectin, we focused on optimizing our method using BSA deposition on tissue culture polystyrene plates.

Next, we rendered the BSA-covered portion of the well cell adherent again by incubating the plate in culture medium supplemented with $10 \mu \mathrm{g} / \mathrm{mL}$ fibronectin. A solution of cell type 2 (mCherry expressing ARPE-19 cells) in serum containing medium was then added to generate a patterned co-culture. Similar to the first seeding step, $1 \times 10^{6}$ cells per well were used, ensuring that $100 \%$ surface coverage immediately after seeding (Figure 1F). Cells were given time to adhere to the available surface before removing excess cells by gentle washing with culture medium. Once unadhered cells were removed, a co-culture with a clear boundary between the two cell types was visible (Figure 1G).

We next determined if our method is suitable for other cell types and if it was possible to generate heterotypic co-cultures. We were able to generate heterotypic co-cultures of HUVEC/ARPE-19 (Figure 2A), homotypic co-cultures of BJ cells (Figure 2B), as well as heterotypic cultures of ARPE-19/MDCK (Figure 2C), and ARPE-19/BJs (Figure
2D). Our results show that the method presented here is compatible for use with different cell types, including epithelial, endothelial, and fibroblast cells. Moreover, the patterning was equally successful in established cell lines and primary cells. In most cases, cell type 2 did not adhere to the areas occupied by cell type 1 , resulting in clearly distinct populations (Figure 1, Figure $2 \mathrm{~A}$ and C). For some cell types, however, the second cell type tended to attach to areas covered by the first cell type. This was the case when BJs were used as cell type 2. In homotypic co-culture of GFP-BJ and mCherry-BJ (Figure 2C), the adhesion of cell type 2 onto cell type 1 was more pronounced than in the heterotypic co-culture of GFP-ARPE-19 and mCherry-BJ (Figure 2D). This suggests the effect is cell type-dependent and can be stronger or weaker for different cell combinations. We speculate that cells which readily form aggregates will be more challenging to pattern as they will tend to adhere equally to both the exposed TCPS surface and the pre-existing confluent sheet of cell type 1. For this reason, when generating heterotypic co-cultures, if one cell type is particularly adhesive to either itself or the other cell type, this cell population should be patterned in the first seeding step to ensure optimal pattern quality.

Certain applications (12) may require prolonged maintenance of patterned co-cultures. To test whether our technology is suitable for these applications, we maintained co-cultures for seven days, finding patterns were extremely stable; even after one week in culture, the original boundary between the two cell populations was clearly visible (Figure 2E-H). This was true for homotypic (Figure $2 \mathrm{H}$ ) as well as heterotypic co-cultures (Figure 2F). We quantified the extent of pattern disruption for a homotypic co-culture (Supplementary Figure S4). Pattern disruption is significantly less than what was observed using microcontact printing with the same combination of cell types (22). This limited cell-cell mixing behavior is potentially useful for multi-day experiments where the extent of cell-cell contact between the two cell types could change signaling properties and hence cellular function. Future work will include more detailed characterization of the pre-patterned surfaces to elucidate the mechanism responsible for the increased stability of patterns generated using the present method.

Depending on the specific application, it may also be desirable to generate co-cultures in wells of different sizes. Therefore, we assessed the compatibility of our method with different well sizes. We attempted to pattern co-cultures in 6-, 12-, 24-, 48-, and 96-well plates. We focused on generating robust patterns at the first cell seeding stage, because we found in our 24-well plate system that this step was the most critical for successful patterning. We were able to successfully pattern cell localization in 6-, 12-, 24-, and 48-well plates (Figure 3A-H). For the 96-well plate size, successful cell patterns could be generated (Figure 3I and J) using a higher concentration of BSA and a higher angle of tilting to make sure the two fronts formed by the meniscus of BSA solution did not touch. We verified that the higher BSA concentration in the pre-patterning step did not negatively affect the outcome of co-culture in 96-well plates (Supplementary Figure S5). We are confident our method can produce reliable patterning in wells of 96 -well plates and larger.

It should be noted that with decreasing well size, we found that the contour of the pattern became more curved. In 6-well plates, approximately $50 \%$ of the interface was straight; however, in smaller wells the BSA solution generated a curved interface (Figure 3G). The curvature of the interface results from meniscus formation when BSA solution is placed in a well. In the case of 6-well plates, the contribution of the meniscus to the final shape is negligible. However, in smaller wells the meniscus effect is so large that the two fronts of BSA solution join at the top of the well, resulting in an oval-shaped interface as opposed to a semicircle. We expect that supplementing the BSA solution with a cell culture-compatible surfactant (23) or changing the tilt angle (as used in the 96-well plate case) will enable the generation of straighter interfaces.

Among the most important requirements for assays designed for highthroughput applications are consistency and reproducibility. For example, it is important that patterning in all of the wells of an individual plate 

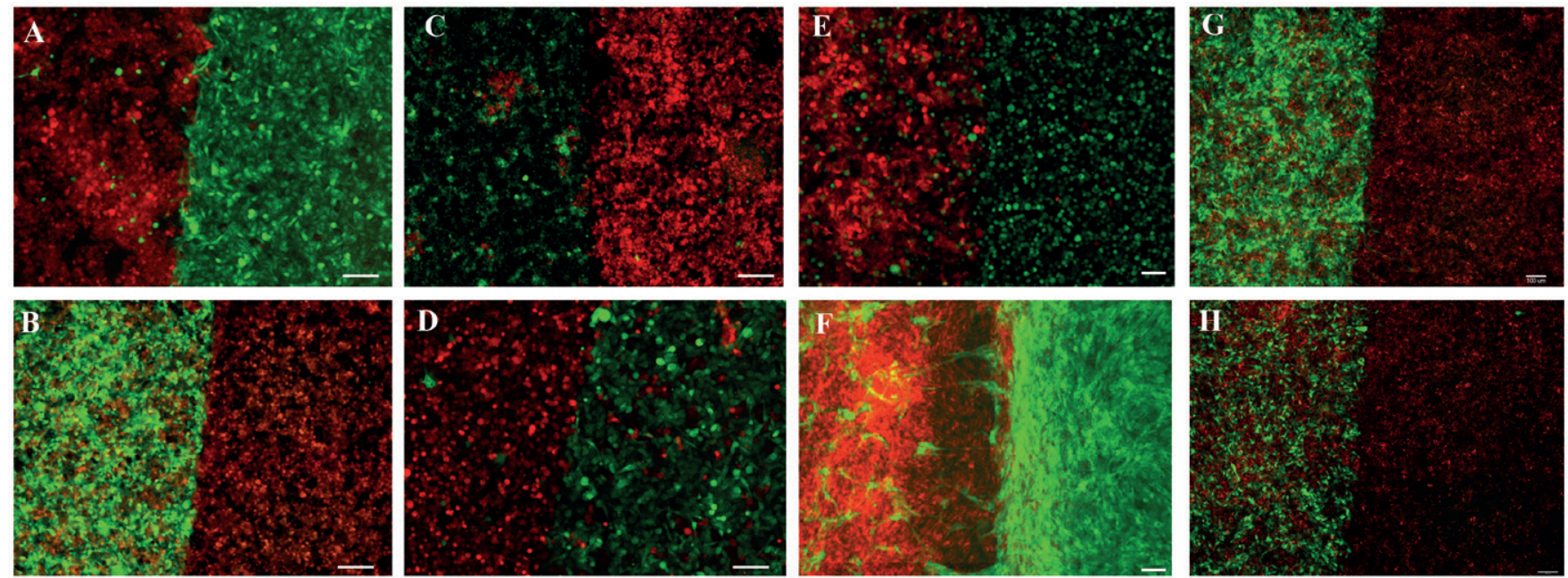

Figure 2. Patterned co-cultures. (A) Co-culture of GFP-HUVECs (green) and mCherry-ARPE-19 (red). (B) Co-culture of GFP-BJ (green) and mCherry-BJ cells (red). (C) Co-culture of GFPE-ARPE-19 (green) and mCherry-MDCK (red). (D) Co-culture of GFP-ARPE-19 (green) and mCherry-BJ cells (red). Images in A, B, C, and D were taken $12 \mathrm{~h}$ after pattern generation. (E) Co-culture of GFP-ARPE-19 (green) and mCherry-3T3 cells (red) one day after patterning. (F) Co-culture of GFP-ARPE-19 (green) and mCherry-3T3 cells (red) seven days after patterning. (G) Co-culture of GFP-BJ (green) and mCherry-BJ cells (red) one day after patterning. (H) Co-culture of GFP-BJ (green) and mCherry-BJ cells (red) seven days after patterning. All scale bars are 100 um.

in parallel is successful. To better characterize the reliability of our patterning, we measured the reproducibility of interface generation at the pre-patterning and cell patterning steps. Analysis of the reproducibility of our protein pre-patterning step (Supplementary Figure S6A) shows that by tilting the plate we were able to ensure reproducible BSA adsorption in all wells of the plate. Furthermore, we verified that the tilting of the plate does not result in uneven protein adsorption in different locations of the pre-patterned surface (Supplementary Figure S6B). We next attempted simultaneous patterning of cells in all wells of a 24-well plate using ARPE-19 cells to characterize the consistency of the cell patterning step. We were able to successfully generate cell patterns in all 24 wells, demonstrating the consistency of our method.
Reproducible patterning is also particularly important in high-throughput imaging applications where low variation in the positioning of the interface between the two cell types allows the interface region to be captured in a single image obtained at the same position from well to well, preventing the need for cumbersome post-acquisition image analysis. To test whether our method produced sufficiently reproducible patterning for high-content imaging, we assessed the spatial reproducibility of our pattern. Specifically, we quantified the well-towell variation in the location of the interface of the patterned cell populations. For each well, we measured the y-coordinates of the interface at set values of $\mathrm{x}$-coordinates. Figure $4 \mathrm{~A}$ shows the results of this analysis in coordinates converted into distance from the bottom/ right corner of the screen. Deviations in the position of interface in different wells were minimal with the relative standard deviation in different locations ranging from $0.6 \%$ to $9.1 \%$, corresponding to a displacement of less than $100 \mu \mathrm{m}$ at the center point of the interface. This deviation easily allows for capturing the interface in the same image frame location in each well at a $4 \times$ magnification. To demonstrate this, we overlaid images of the interface with the closest representation of the average localization against two images representing the localization of interfaces with the most deviation from the average (Figure 4B). All three interfaces are easily captured with the same image frame location using automated microscopy. This is significant because existing patterning methods often have an imperfect success rate

\section{6-well}
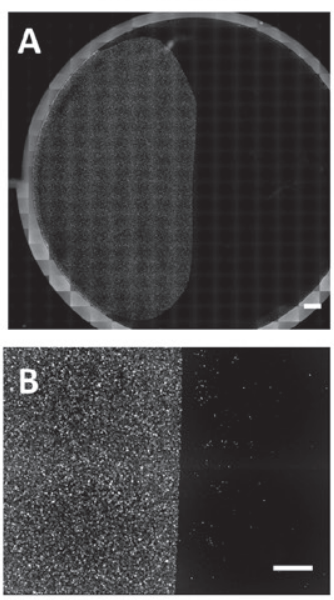

12-well
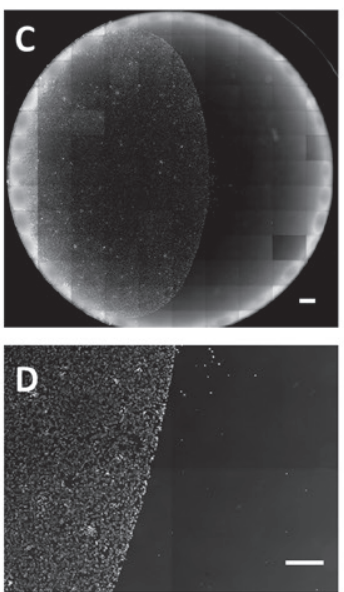

24-well
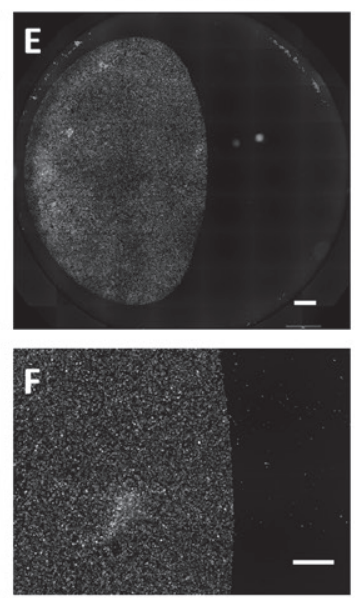

48-well
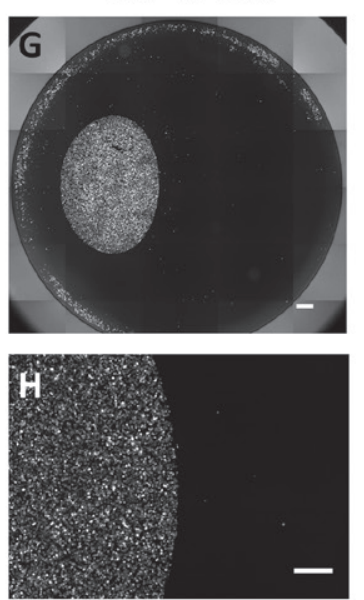

96-well
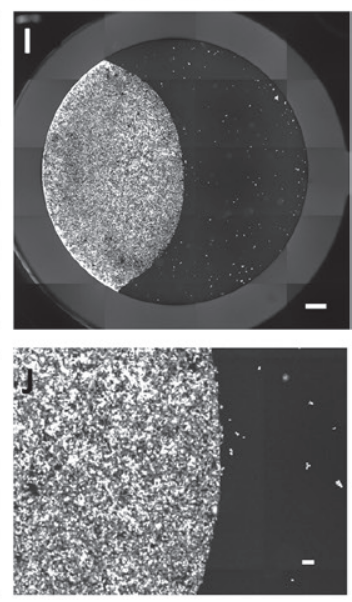

Figure 3. Patterning cells in wells of different sizes. (A) Cell pattern generated in a 6 -well plate. Scale bar is $2 \mathrm{~mm}$. (B) Close-up of cell pattern generated in a 6-well plate. Scale bar is $500 \mu \mathrm{m}$. (C) Cell pattern generated in a 12-well plate. Scale bar is $1 \mathrm{~mm}$. (D) Close-up of cell pattern generated in a 12 -well plate. Scale bar is $500 \mu \mathrm{m}$. (E) Cell pattern generated in a 24-well plate. Scale bar is $1 \mathrm{~mm}$. (F) Close-up of cell pattern generated in a 24-well plate. Scale bar is $500 \mu \mathrm{m}$. (G) Cell pattern generated in a 48 -well plate. Scale bar is $500 \mu \mathrm{m}$. (H) Close-up of cell pattern generated in a 48 -well plate. Scale bar is $400 \mu \mathrm{m}$. (I) Cell pattern generated in a 96-well plate. Scale bar is $500 \mu \mathrm{m}$. (J) Close-up of cell pattern generated in a 96-well plate. Scale bar is $100 \mu \mathrm{m}$. Light areas indicate the presence of GFP-ARPE19 cells and dark areas indicate that no cells are present. 
and positioning the pattern in the samelocation in every well requires careful alignment of the stamp or stencil within a well.

To demonstrate the utility of generating a defined interface between the two cell types, a co-culture of wild type MDCK and MDCK cells overexpressing GFP-N-cadherin (Supplementary Figure S7A) was studied. At the pattern interface, GFP-N-cadherin MDCK cells are surrounded on one side with wild type cells and on the other side by self-like GFP-Ncadherin cells. Interestingly, we noticed that in these interface cells GFP-N-cadherin was preferentially enriched on the side of the cells in contact with other GFP-N-cadherin overexpressing cells. We confirmed this asymmetric localization of GFP-N-cadherin by quantifying GFP signal intensity at the membrane on either side of the cells at the interface (Supplementary Figure S7A). The planar polarization of the GFP-tagged protein is consistent with homotypic inter-cellular interactions of $\mathrm{N}$-cadherin (24) between neighboring cells. This specific model system could be useful for screening for molecules involved in this intercellular signaling process.

We present for the first time a method for medium-throughput generation of 2-D patterned co-cultures. The simplicity and reliability of our method, combined with its compatibility with high-content microscopy, make it a potentially useful tool for generating a more in vivo-like cell culture platform for screening applications.

\section{Acknowledgments}

We acknowledge R. Sodi and SI Ontario for technical assistance. This work was funded by a Natural Science and Engineering Research Council of Canada (NSERC) Discovery grant and a Connaught Early Researcher Award to A.M. and an NSERC graduate scholarship and CIHR Training Program in Regenerative Medicine Fellowship to S.J. The authors have no conflict of interests to declare. S.J. and K.L. designed the project, conducted experiments, analyzed the data and wrote the manuscript, A.M. designed the project, analyzed the data, and wrote the manuscript.

\section{Competing interests}

The authors declare no competing interests.

\section{References}

1. Herzog, E.L. and R. Bucala. 2010. Fibrocytes in health and disease. Exp. Hematol. 38:548-556.

2. Nguyen, D.X., P.D. Bos, and J. Massague. 2009. Metastasis: from dissemination to organ-specific colonization. Nat. Rev. Cancer 9:274-284.

3. Whitesides, G.M., E. Ostuni, S. Takayama, X. Jiang, and D.E. Ingber. 2001. Soft lithography in biology and biochemistry. Annu. Rev. Biomed. Eng. 3:335-373.
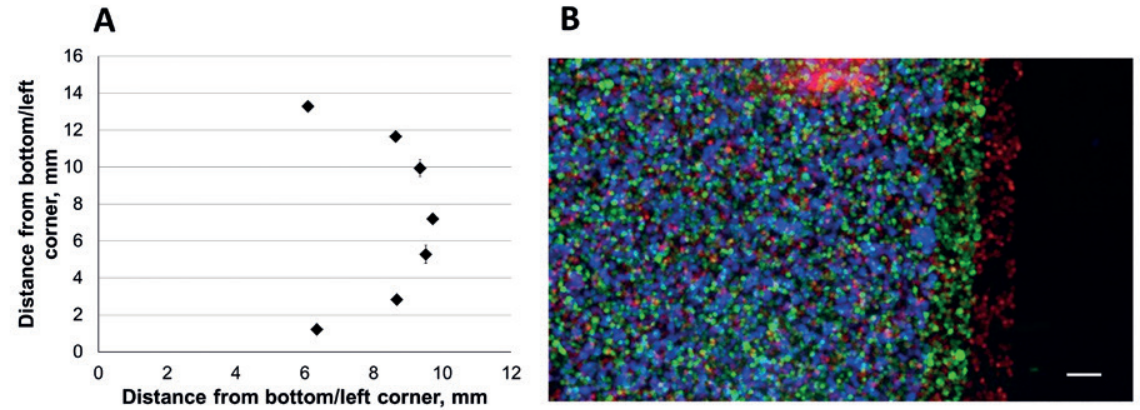

Figure 4. Reproducibility of patterning. (A) Average location of the cell pattern interface in wells from a 24-well plate. For each $\mathrm{x}$-axis measurement, an average of $24 \mathrm{y}$-axis measurements is represented. Error bars represent standard deviation, $n=24$. (B) An overlay of an interface most closely representing the average outcome (green) and the two interfaces with the greatest deviation from the average outcome (red and blue). The scale bar is $100 \mu \mathrm{m}$.

4. Bhatia, S.N., M.L. Yarmush, and M. Toner. 1997. Controlling cell interactions by micropatterning in co-cultures: hepatocytes and 3T3 fibroblasts. J. Biomed. Mater. Res. 34:189-199.

5. Jinno, S., H.C. Moeller, C.L.Chen, B. Rajalingam, B.G. Chung, M.R. Dokmeci, and A. Khademhosseini. 2008. Microfabricated multilayer parylene$\mathrm{C}$ stencils for the generation of patterned dynamic co-cultures. J. Biomed. Mater. Res. A 86:278-288.

6. Wallace, C.S. and G.A. Truskey. 2010. Directcontact co-culture between smooth muscle and endothelial cells inhibits TNF-alpha-mediated endothelial cell activation. Am. J. Physiol. Heart Circ. Physiol. 299:H338-H346.

7. Bhatia, S.N., U.J. Balis, M.L. Yarmush, and M. Toner. 1998. Probing heterotypic cell interactions: hepatocyte function in microfabricated co-cultures. J. Biomater. Sci. Polym. Ed. 9:1137-1160.

8. Bhatia, S.N., U.J. Balis, M.L. Yarmush, and M. Toner. 1999. Effect of cell-cell interactions in preservation of cellular phenotype: cocultivation of hepatocytes and nonparenchymal cells. FASEB J. 13:1883-1900.

9. Truskey, G.A. 2010. Endothelial Cell Vascular Smooth Muscle Cell Co-Culture Assay For High Throughput Screening Assays For Discovery of Anti-Angiogenesis Agents and Other Therapeutic Molecules. Int J High Throughput Screen. 2010:171-181.

10.Shearer, M.C. and J.W. Fawcett. 2001. The astrocyte/meningeal cell interface--a barrier to successful nerve regeneration? Cell Tissue Res. 305:267-273.

11. Cho, C.H., J. Park, A.W. Tilles, F. Berthiaume, M. Toner, and M.L. Yarmush. 2010. Layered patterning of hepatocytes in co-culture systems using microfabricated stencils. Biotechniques 48:47-52.

12. Paz, A.C., S. Javaherian, and A.P. McGuigan. 2012. Micropatterning Co-cultures of Epithelial Cells on Filter Insert Substrates. Journal of Epithelial Biology \& Pharmacology:77-85.

13. Khademhosseini, A., K.Y. Suh, J.M. Yang, G. Eng, J. Yeh, S. Levenberg, and R. Langer. 2004. Layer-by-layer deposition of hyaluronic acid and poly-L-lysine for patterned cell co-cultures. Biomaterials 25:3583-3592.

14. Javaherian, S., K.A. O'Donnell, and A.P. McGuigan. 2011. A fast and accessible methodology for micro-patterning cells on standard culture substrates using Parafilm inserts. PLoS ONE 6:e20909.

15. Peerani, R., C. Bauwens, E. Kumacheva, and P.W. Zandstra. 2009. Patterning mouse and human embryonic stem cells using micro-contact printing. Methods Mol. Biol. 482:21-33.
16. Fernandes, T.G., M.M. Diogo, D.S. Clark, J.S. Dordick, and J.M. Cabral. 2009. High-throughput cellular microarray platforms: applications in drug discovery, toxicology and stem cell research. Trends Biotechnol. 27:342-349.

17. Lee, M.Y., R.A. Kumar, S.M. Sukumaran, M.G. Hogg, D.S. Clark, and J.S. Dordick. 2008. Three-dimensional cellular microarray for highthroughput toxicology assays. Proc. Natl. Acad. Sci. USA 105:59-63.

18. Chung, S., R. Sudo, V. Vickerman, I.K. Zervantonakis, and R.D. Kamm. 2010. Microfluidic platforms for studies of angiogenesis, cell migration, and cell-cell interactions. Sixth International Biol.-Fluid Mechanics Symposium and Workshop March 28-30, 2008 Pasadena, California. Ann. Biomed. Eng. 38:1164-1177.

19. Tumarkin, E., L. Tzadu, E. Csaszar, M. Seo, H. Zhang, A. Lee, R. Peerani, K. Purpura, et al. 2011. High-throughput combinatorial cell co-culture using microfluidics. Integr Biol (Camb). 3:653662.

20. Jeyachandran, Y.L., E. Mielczarski, B. Rai, and J.A. Mielczarski. 2009. Quantitative and qualitative evaluation of adsorption/desorption of bovine serum albumin on hydrophilic and hydrophobic surfaces. Langmuir 25:11614-11620.

21. Kowalczyńska, H.M., M. Nowak-Wyrzykowska, A.A. Szczepankiewicz, J. Dobkowski, M. Dyda, J. Kaminski, and R. Kolos. 2011. Albumin adsorption on unmodified and sulfonated polystyrene surfaces, in relation to cell-substratum adhesion. Colloids Surf. B Biointerfaces 84:536544.

22. Javaherian, S., N. Anesiadis, R. Mahadevan, and A.P.McGuigan. 2013. Design principles for generating robust gene expression patterns in dynamic engineered tissues. Integr Biol (Camb). 5:578-589.

23. Srigunapalan, S., I.A. Eydelnant, C.A. Simmons, and A.R. Wheeler. 2012. A digital microfluidic platform for primary cell culture and analysis. Lab Chip 12:369-375.

24. Derycke, L.D. and M.E. Bracke. 2004. N-cadherin in the spotlight of cell-cell adhesion, differentiation, embryogenesis, invasion and signalling. Int. J. Dev. Biol. 48:463-476.

Received 14 February 2013; accepted 01 May 2013.

Address correspondence to Alison P. McGuigan, Department of Chemical Engineering and Applied Chemistry, University of Toronto, Toronto, Canada. E-mail: alison.mcguigan@utoronto.ca

To purchase reprints of this article, contact: biotechniques@fosterprinting.com 